Rie Matsushita, Keisuke Nagasaki, Tadayuki Ayabe, Yoko Miyoshi, Saori Kinjo, Hidenori Haruna, Kenji Ihara, Tomonobu Hasegawa, Shinobu Ida, Keiichi Ozono, Kanshi Minamitani* and Thyroid Committee of the Japanese Society for Pediatric Endocrinology

\title{
Present status of prophylactic thyroidectomy in pediatric multiple endocrine neoplasia 2: a nationwide survey in Japan 1997-2017
}

https://doi.org/10.1515/jpem-2018-0444

Received October 15, 2018; accepted February 8, 2019; previously published online May 31, 2019

\section{Abstract}

Background: In Japan, prophylactic thyroidectomy involves out-of-pocket expense. The American Thyroid Association (ATA) recommends prophylactic thyroidectomy for medullary thyroid carcinoma (MTC) during early childhood in patients with multiple endocrine neoplasia type 2 (MEN2). The ATA reports a high frequency of postoperative complications in childhood, which also influenced the delay of prophylactic thyroidectomy in Japan.

Methods: This retrospective study of multiple medical centers in Japan included individuals aged $<20$ years diagnosed with germline RET mutations between 1997 and 2017. The onset and onset possibility were defined based on confirmed lesions or calcitonin levels. The definition of risk and prophylactic thyroidectomy were based on the ATA 2015 revised guideline.

Results: Twenty-one patients with MEN2 were enrolled (highest risk, $\mathrm{n}=5$; high risk, $\mathrm{n}=5$; and moderate risk, $\mathrm{n}=11)$. The cumulative incidence of the onset/onset possibility reached $50 \%$ at 5 and 8 years and $100 \%$ at 9 years

*Corresponding author: Kanshi Minamitani, MD, PhD, Department of Pediatrics, Teikyo University Chiba Medical Center, 3426-3

Anesaki, Ichihara-shi, Chiba 299-0111, Japan,

Phone: +81-4-3662-1211, Fax +81-4-3661-2175,

E-mail:kminami@med.teikyo-u.ac.jp

Rie Matsushita: Division of Pediatrics, Kikugawa General Hospital, Shizuoka, Japan. https://orcid.org/0000-0003-3328-9499; and Department of Pediatrics, Hamamatsu University School of Medicine, Shizuoka, Japan

Keisuke Nagasaki: Division of Pediatrics, Department of Homeostatic Regulation and Development, Niigata University Graduate School of Medical and Dental Sciences, Niigata, Japan

Tadayuki Ayabe: Japan Environment and Children's Study Medical Support Center, National Research Institute for Child Health and Development, Tokyo, Japan and 17 years in high- and moderate-risk patients, respectively. Of 7 patients with MEN2A, 71\% underwent prophylactic thyroidectomy. Only one 5-year-old patient (C634Y) had increased serum calcitonin level after prophylactic thyroidectomy in the MEN2A group. The only permanent complication, which did not occur in patients who underwent total thyroidectomy alone, was hypoparathyroidism (33\% of patients). This permanent complication occurred with clinically developed MTC. No permanent postoperative complications occurred in patients aged 5-6 years.

Conclusions: Prophylactic thyroidectomy reduces recurrence and postoperative complications in pediatric patients with MEN2. Early thyroidectomy based on only calcitonin level could possibly reduce thyroidectomy delay.

Keywords: central node dissection; medullary thyroid carcinoma; multiple endocrine neoplasia type 2; postoperative complications; prophylactic thyroidectomy; recurrence.

\section{Introduction}

Medullary thyroid carcinoma (MTC) arises from $C$ cells and occurs in almost all patients with multiple endocrine neoplasia type 2 (MEN2) caused by germline mutations in the

Yoko Miyoshi and Keiichi Ozono: Department of Pediatrics, Osaka University Graduate School of Medicine, Osaka, Japan Saori Kinjo: Division of Pediatrics, Okinawa Chubu Hospital, Okinawa, Japan

Hidenori Haruna: Department of Pediatrics and Adolescent Medicine, Juntendo University Graduate School of Medicine, Tokyo, Japan Kenji Ihara: Department of Pediatrics, Faculty of Medicine, Oita University, Oita, Japan

Tomonobu Hasegawa: Department of Pediatrics, Keio University School of Medicine, Tokyo, Japan

Shinobu Ida: Department of Gastroenterology and Endocrinology, Osaka Women's and Children's Hospital, Osaka, Japan; and Pediatric Chronic Intestinal Pseudo-Obstruction (CIPO), Research Group for "Comprehensive Study and Seamless Guidelines" on Rare and Intractable Gastrointestinal Disease from Childhood, Supported by the Ministry of Health, Labor and Welfare of Japan, Tokyo, Japan 
RET gene. Because lymph node metastasis starting during the early stage of tumorigenesis is common, patients with MTC are at increased risk of postoperative recurrence [1]. Moreover, in hereditary MTC, the rate of progression from C cell hyperplasia (CCH) to MTC increases with age [2].

For hereditary MTC, prophylactic total thyroidectomy in early childhood is recommended by a consensus statement by Brandi et al. and the guidelines established by the American Thyroid Association (ATA), according to the estimated recurrence risk [3-5], and is an important treatment option for recurrence-free survival in all carriers of RET gene mutations [3-5]. Based on genotype-phenotype correlations, ATA classified the risks of hereditary MTC as highest (RET M918T mutation, phenotype MEN2B), high (codon 634 mutations, phenotype MEN2A and RET A883F mutation, phenotype MEN2B), and moderate (RET codon mutations other than M918T, C634, and A883F, phenotype MEN2A) in the 2015 revised guidelines for the diagnosis and management of patients with MTC [5].

However, the ATA also reports that thyroid surgery frequently causes postoperative complications in children, particularly in infants [5, 6]; the most common postoperative complications are hypoparathyroidism [7-9] and recurrent laryngeal nerve (RLN) palsy.

Due to concerns about balancing the need for prophylactic resection and overtreatment/postoperative complications, thyroid surgery is often performed at age $>10$ years in Japan [10, 11]. Moreover, in Japan, RET gene testing during screening of patients with MTC is covered by insurance, but RET screening test before onset in the proband's family and prophylactic thyroidectomy are out-of-pocket expenses. Surgery at a higher age than the ATA-recommended age may be the cause of the $39 \%$ recurrence at age $10-20$ years among Japanese pediatric hereditary MTC patients [10, 11].

It remains unclear whether the low rate of complications from thyroid surgery in early childhood reported in large facilities is also true when a smaller number of pediatric patients with thyroid cancer are treated. Thyroid cancer rarely occurs in children, with an incidence of 0.54 per 100,000 person-years. Furthermore, MTC accounts for only $5 \%$ of all pediatric thyroid cancer patients [12]. Therefore, few studies concerning postoperative complications of prophylactic thyroidectomy in children with MEN2 have been reported from large facilities [7-9].

The introduction of a sensitive diagnostic assay using calcitonin as a tumor marker for MTC has increased the accuracy of predicting malignancy [13]. Electrochemiluminescence immunoassay (ECLIA) is currently considered the most sensitive diagnostic test for MTC [14] and has been used to measure calcitonin in all medical facilities in Japan since April 2015. Herein, we aimed to identify the pathological onset or timing of increased calcitonin levels among Japanese children, the frequency of prophylactic thyroidectomy, prognosis, complications and rate of recurrence in patients with hereditary MTC. Toward this goal, the Japanese Society for Pediatric Endocrinology (JSPE) conducted a nationwide survey of young patients with RET gene mutations or MEN2.

\section{Subjects and methods}

\section{Inclusion and exclusion criteria}

Participants included patients aged $<20$ years diagnosed with MEN2 or an RET gene mutation between January 1997 and January 2017. Patients were excluded if they [1] did not consent to participate in this study, [2] did not undergo a genetic test, or [3] had neither an operative record nor a record of calcitonin levels. The study was conducted in accordance with the tenets of the Declaration of Helsinki. The research protocol was approved by the Ethics Committee of the Hamamatsu University School of Medicine (\#E16-230) and the ethics committees of the facilities of the co-authors. Written informed consent was obtained from all participants or their parents or guardians. Four patients who were lost to follow-up were considered as "opted out".

\section{Research design and setting}

The study design was a survey of medical records. We reviewed clinical records and mailed a questionnaire (including questions on the subjects' sex, age at diagnosis and at thyroidectomy, duration of follow-up, genetic testing, phenotype, calcitonin values over time, dates of calcitonin measurements, surgical interventions, node dissection area, pathological diagnoses, postoperative complications, type of surgeon and facility [own vs. other], and outcomes) to 190 councilors of the JSPE, pediatricians and pediatric surgeons who submitted data on MEN2 to the Research Group for "Comprehensive Study and Seamless Guidelines" on rare and intractable gastrointestinal diseases of childhood supported by the Japanese Ministry of Health, Labor and Welfare.

\section{Calcitonin testing}

In Japan, radioimmunoassay (RIA [using the LSIM $^{\circledR}$ Calcitonin test system, LSI Medience, Tokyo, Japan) was used to evaluate calcitonin levels until March 2015. In the RIA, the reference values for basal and stimulated levels for calcitonin are $<86 \mathrm{pg} / \mathrm{mL}$ [15] and $<300 \mathrm{pg} / \mathrm{mL}$, respectively [16]. The ECLIA (using the ECLusys ${ }^{\circledast}$ Calcitonin test system, Roche Diagnostics, Mannheim, Germany) has been used since April 2015. In this test, the reference values of basal calcitonin are $<3.91 \mathrm{pg} / \mathrm{mL}$ in women and $<5.15 \mathrm{pg} / \mathrm{mL}$ in men [15], and those of the stimulated level are $<67.6 \mathrm{pg} / \mathrm{mL}$ in women and $<83.7 \mathrm{pg} / \mathrm{mL}$ in men $[17,18]$. The lower limit of detection is $0.5 \mathrm{pg} / \mathrm{mL}$. Calcitonin stimulation testing is performed as follows: calcium $25 \mathrm{mg} / \mathrm{kg}$ is intravenously injected, after which blood is collected at 0 (pre), 2, 5, and 10 min [19]. Only Roche's Japanese agency handled the calcitonin 
ECLIA reagent and all institutes included in this study measured calcitonin via the ECLIA after April 2015.

\section{Definition of prophylactic thyroidectomy}

In this study, prophylactic thyroidectomy was defined as removal of the thyroid before MTC development or while MTC was clinically unapparent and confined to the gland. This definition was based on the following ATA specifications: resection performed within a year of birth in the highest-risk patients, resection performed at the age of 5 years or younger depending on calcitonin levels in high-risk patients, and resection performed when calcitonin levels were elevated or the patient's parents preferred a resection and did not desire long-term active surveillance in moderate-risk patients [5].

\section{Definitions of disease onset or first increased serum calcitonin levels and recurrence, persistent disease or residual thyroid tissue}

Disease onset was defined as the time when MTC or CCH was pathologically diagnosed. First increased serum calcitonin levels were defined as the time when the calcitonin levels in the stimulation test exceeded $300 \mathrm{pg} / \mathrm{mL}$ for patients followed-up through calcitonin measurements via RIA, or when the basal value first exceeded the adult reference value for patients followed via ECLIA. Because the basal calcitonin level is physiologically high in patients aged $<3$ years $[20,21]$, first increased serum calcitonin level in these patients was defined as the time when both basal and stimulated values simultaneously exceeded the adult baseline level.

The evaluation term of patients following thyroidectomy was based on the ATA revised guidelines [5]. Biochemical recurrence or persistence was defined as a calcitonin level $\geq 0.5 \mathrm{pg} / \mathrm{mL}$ via ECLIA more than 6 months after total thyroidectomy. When calcitonin level was measured after total thyroidectomy, additional evaluation for residual tissue and thyroglobulin measurement was performed. Clinical recurrence or persistence was defined as tumors diagnosed via imaging after treatment or pathologically-diagnosed MTC after second surgery.

\section{Statistical analysis}

Kaplan-Meier analysis was used to evaluate the association between disease onset and age. Competing risks resulting from prophylactic thyroidectomy were assumed using the Weibull parameters by adjustment with exclusion. For the analysis of postoperative complications, we used descriptive statistics and prepared a cross table of the age at surgery and the level of neck lymphadenectomy. JMP version 8.00 (SAS Institute, Cary, NC) was used for all analyses. Statistical significance was set at $\mathrm{p}<0.05$.

\section{Results}

Of the 26 patients registered by the Thyroid Committee of the JSPE, 21 patients with MEN2 were enrolled (highest risk, $\mathrm{n}=5$; high risk, $\mathrm{n}=5$; and moderate risk, $\mathrm{n}=11$ ) (Figure 1). Patient characteristics are shown in Table 1. All 16 patients with MEN2A, i.e. those with a high or moderate risk, had a family history of MEN2 and had undergone genetic testing at a young age (median, 3 years; range, $0-8$ years). All patients with MEN2B,
14 patients (MEN2A, $n=12$; MEN2B, $n=2$ ) belonged to 14 families identified through JSPE councilor screening (99 facilities, 151 councilors, the response rate $79.5 \%$ )

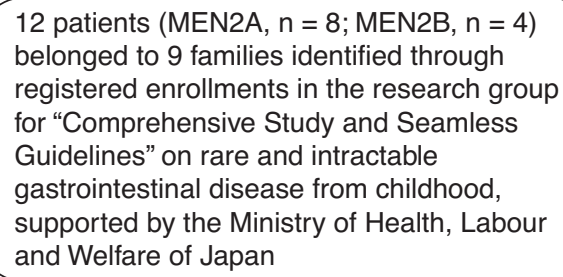

1 MEN2B patient registered at 2 facilities

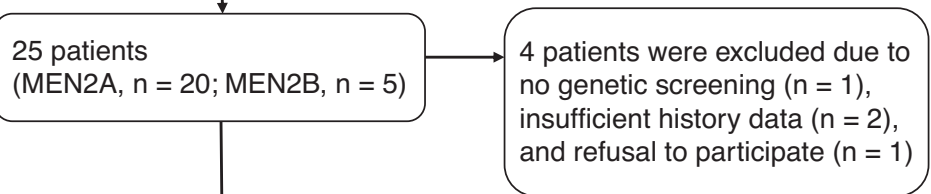

21 patients (MEN2A, $n=16$; MEN2B, $n=5$ ) belonging to 18 families (4 moderate-risk patients belonged to the same family) were included

Figure 1: Inclusion and exclusion criteria. 
Table 1: Demographic and clinical characteristics of the patients

\begin{tabular}{|c|c|c|c|c|c|c|c|c|c|c|c|}
\hline \multirow[t]{3}{*}{$\begin{array}{l}\text { Patient } \\
\text { no. }\end{array}$} & \multirow[t]{3}{*}{ Sex } & \multirow[t]{3}{*}{$\begin{array}{l}\text { RET } \\
\text { mutation }\end{array}$} & \multicolumn{3}{|c|}{$\begin{array}{l}\text { Age at genetic } \\
\text { screening (I), } \\
\text { pathological onset } \\
\text { or first calcitonin } \\
\text { increase timing (II), } \\
\text { thyroidectomy (III) }\end{array}$} & \multicolumn{2}{|c|}{$\begin{array}{l}\text { Calcitonin level at } \\
\text { first increase or at } \\
\text { thyroidectomy ECLIA } \\
\text { RIA* }^{\text {a }}\end{array}$} & \multirow[t]{3}{*}{$\begin{array}{l}\text { TNM stage or } \\
\text { pathological } \\
\text { findings/Node } \\
\text { dissection }\end{array}$} & \multicolumn{2}{|c|}{$\begin{array}{l}\text { Postoperative } \\
\text { calcitonin level } \\
\text { ECLIA RIA* }\end{array}$} & \multirow{3}{*}{$\begin{array}{l}\text { Duration of } \\
\text { follow-up } \\
\text { (from } \\
\text { onset) } \\
\text { Years }\end{array}$} \\
\hline & & & I & II & III & Basal & Peak & & Basal & Peak & \\
\hline & & & \multicolumn{3}{|c|}{ Years } & \multicolumn{2}{|l|}{$\mathrm{pg} / \mathrm{mL}$} & & \multicolumn{2}{|l|}{$\mathrm{pg} / \mathrm{mL}$} & \\
\hline $1^{\S}$ & $M$ & $\mathrm{C} 634 \mathrm{Y}$ & 0 & 5 & 5 & $71^{*}$ & $428^{*}$ & $\begin{array}{l}\mathrm{CCH} \\
/ \text { Not performed }\end{array}$ & 1.33 & NT & 2 \\
\hline 2 & $\mathrm{~F}$ & C634G & 0 & 8 & 9 & 11.3 & 333 & $\begin{array}{l}\text { T1aNomo } \\
\text { /Central }\end{array}$ & $<0.5$ & NT & 1.1 \\
\hline 3 & $\mathrm{~F}$ & C634S & 8 & 9 & 10 & $72^{*}$ & $670^{*}$ & $\begin{array}{l}\text { T1aNOMO } \\
\text { /Central }\end{array}$ & $<0.5$ & NT & 3.3 \\
\hline 4 & $F$ & C634R & 1 & 5 & & 18.6 & 148 & & & & $(0.5)$ \\
\hline 5 & $F$ & $\mathrm{C} 634 \mathrm{Y}$ & 0 & 2 & & 17.9 & 187 & & & & (1.8) \\
\hline 6 & $\mathrm{~F}$ & C620 & 4 & 17 & 17 & $28^{\star}$ & $110^{\star}$ & $\begin{array}{l}\text { TxNxMO } \\
\text { /Not performed }\end{array}$ & $<0.5$ & NT & 7.8 \\
\hline 7 & $M$ & C620R & 5 & & 10 & $41^{*}$ & $43^{*}$ & $\begin{array}{l}\text { NED } \\
\text { /Not performed }\end{array}$ & $<0.5$ & NT & 3.1 \\
\hline 8 & $\mathrm{~F}$ & C620 & 6 & 6 & 6 & $29^{\star}$ & NT & $\begin{array}{l}\text { T1N0M0 } \\
\text { /Central }\end{array}$ & $<0.5$ & NT & 2.4 \\
\hline 9 & $\mathrm{~F}$ & C620 & 0 & & 5 & $34^{*}$ & NT & $\begin{array}{l}\text { NED } \\
\text { /Central }\end{array}$ & $<0.5$ & NT & 7.8 \\
\hline 10 & $M$ & C618S & 5 & 8 & & 6.8 & NT & & & & $(1.8)$ \\
\hline 11 & $\mathrm{~F}$ & C620Y & 2 & 8 & & 7.4 & NT & & & & $(1.0)$ \\
\hline 12 & $\mathrm{~F}$ & C618R & 0 & 2 & & 4.9 & 120 & & & & $(0.8)$ \\
\hline 139 & $\mathrm{~F}$ & C618R & 7 & LOF & & $46^{*}$ & NT & & & & \\
\hline 149 & $F$ & $\mathrm{C} 618 \mathrm{R}$ & 5 & LOF & & $63^{*}$ & NT & & & & \\
\hline 159 & M & C618R & 6 & LOF & & $33^{*}$ & NT & & & & \\
\hline 169 & $M$ & C618R & 0 & LOF & & NT & NT & & & & \\
\hline $17^{\prime \prime}$ & $\mathrm{F}$ & M918T & 12 & UNK & 12 & $12,000^{*}$ & NT & $\begin{array}{l}\text { TxN1M0 } \\
\text { /D2b }\end{array}$ & UNK & & 2.6 \\
\hline $18^{\ddagger}$ & $M$ & M918T & 11 & UNK & 11 & $78^{*}$ & $720^{*}$ & $\begin{array}{l}\text { T1aN1M0 } \\
\text { /Central }\end{array}$ & $52^{*}$ & $300^{*}$ & 2.5 \\
\hline 19 & M & M918T & 12 & UNK & 12 & $380^{*}$ & $>1,600^{*}$ & $\begin{array}{l}\text { T1bNOM0 } \\
\text { /Central, lateral }\end{array}$ & $46^{*}$ & $45^{\star}$ & 0.2 \\
\hline $20^{\S}$ & $M$ & M918T & 13 & UNK & 13 & $>1,600^{\star}$ & NT & $\begin{array}{l}\text { T2N1M0 } \\
\text { /D2b }\end{array}$ & 332 & NT & 5.6 \\
\hline $21^{\ddagger}$ & $\mathrm{F}$ & M918T & 10 & UNK & 10 & $8,870^{*}$ & NT & $\begin{array}{l}\text { T2NOMO } \\
\text { /Central }\end{array}$ & 3.160 & NT & 11.9 \\
\hline
\end{tabular}

ПSame family.

*Calcitonin measurement via the radioimmunoassay.

$\S B$ Biochemical recurrence.

¥Clinical recurrence.

//No clinical recurrence.

Notes: "Basal" denotes plasma calcitonin levels before calcium stimulation; "peak" denotes plasma calcitonin levels after stimulation. Normal calcitonin levels in the radioimmunoassay (RIA) are $\leq 85 \mathrm{pg} / \mathrm{mL}$ (basal) and $300 \mathrm{pg} / \mathrm{mL}$ (peak). Normal calcitonin levels in the electrochemiluminescence immune assay (ECLIA) are $\leq 3.91 \mathrm{pg} / \mathrm{mL}$ (basal) and $67.6 \mathrm{pg} / \mathrm{mL}$ (peak) in girls and $\leq 5.15 \mathrm{pg} / \mathrm{mL}$ (basal) and $83.7 \mathrm{pg} / \mathrm{L}$ (peak) in boys. Patient 17 was followed up for calcitonin at another facility that performed the thyroidectomy; she was followed-up regarding pheochromocytoma at our facility.

Abbreviations: $\mathrm{CCH}, \mathrm{C}$ cell hyperplasia; ECLIA, electrochemiluminescence immune assay; LOF, loss of follow-up; NED, no evidence of disease; NT, not tested; RIA, radioimmunoassay; UNK, unknown.

i.e. those with the highest risk, had a de novo mutation and had been diagnosed at an older age, after the MTC clinically occurred. Of the five patients with MEN2B, four
(80\%) underwent a detailed examination for refractory diarrhea or constipation before being diagnosed with MTC (data not shown). 


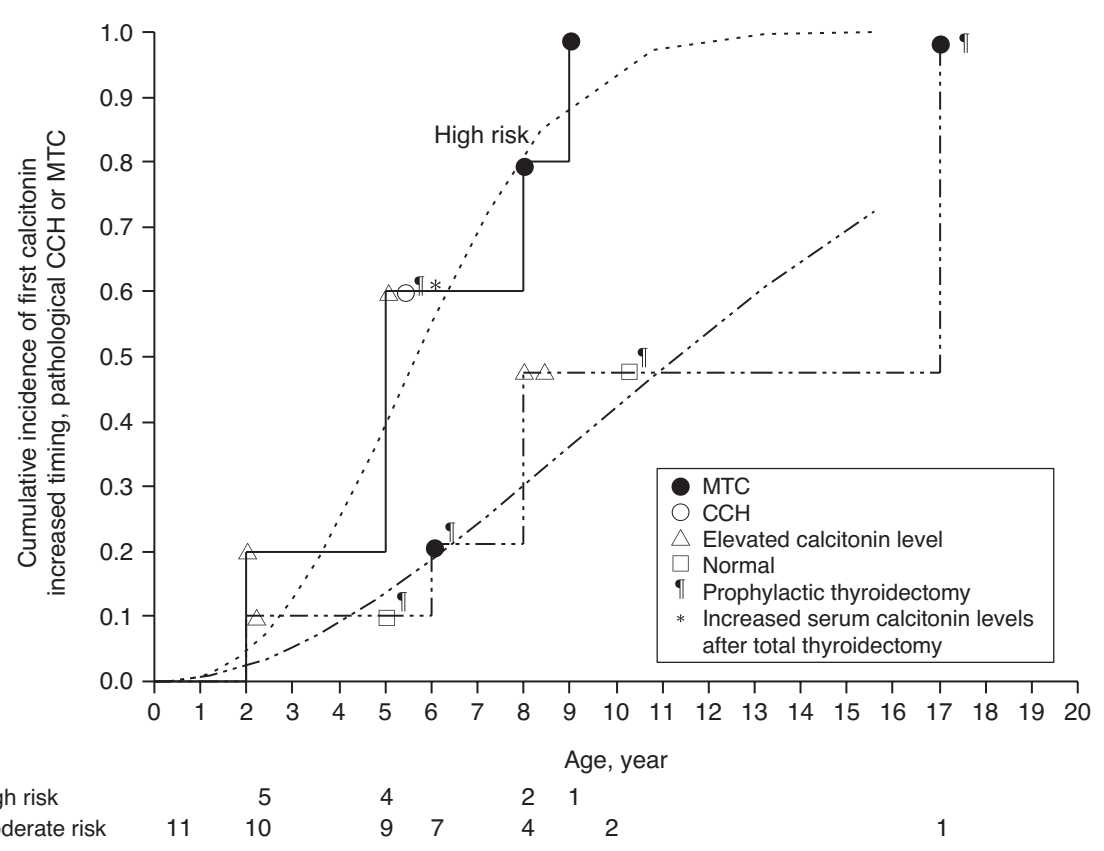

Figure 2: Cumulative incidence of first serum calcitonin increased timing, $\mathrm{C}$ cell hyperplasia (CCH) or medullary thyroid carcinoma (MTC) in patients with multiple endocrine neoplasia type 2A (MEN2A) $(n=16)$.

The solid line indicates high-risk patients $(n=5)$, whereas the dotted line indicates moderate-risk patients $(n=11)$. Each curve represents adjustments for competing risks for high- and moderate-risk patients. The onset in patients with pathological MTC or CCH and the adjusted onset in pathologically normal patients undergoing surgery are shown as follows: $:$ : MTC, O: $\mathrm{CCH}$, and $\square$ : normal. $\triangle$ Patients with increased calcitonin levels who did not undergo surgery. ๆ The need for prophylactic thyroidectomy was determined according to the American Thyroid Association (ATA) definition: high risk, before or at 5 years of age based on serum calcitonin levels; moderate risk, serum calcitonin level increase or children of parents who refused long-term treatment. *Increased serum calcitonin levels after total thyroidectomy was defined as an increase in baseline calcitonin levels of $>0.5 \mathrm{pg} / \mathrm{mL}$ after total thyroidectomy, as measured via the electrochemiluminescence immunoassay (ECLIA). The baseline level of this patient was $1.33 \mathrm{pg} / \mathrm{mL}$.

Pre-symptomatic thyroidectomy was performed in seven of 16 patients with MEN2A (44\%); five patients were awaiting surgery after disease onset possibility (median, 1.0 [range, 0.5-1.8] year), and four patients in the same family were lost to follow-up. Five of the seven patients (71\%) with MEN2A who underwent surgery received prophylactic thyroidectomy as defined by the ATA. No thyroid lesion was detected preoperatively in any patient with MEN2A.

The dates of pathological disease onset or first increased calcitonin timing, surgical pathological diagnosis, prognosis, and rate of recurrence or residual disease or residual thyroid tissues in patients with MEN2A are shown in Figure 2.

\section{ATA high-risk patients}

Calcitonin measurements via ECLIA showed that the mean disease onset possibility was at 5 years of age in ATA high-risk patients. The cumulative incidence of pathological onset or first calcitonin elevation timing reached $50 \%$ at the age of 5 years and $100 \%$ at the age of
9 years (Figure 2). Patient no. 1 underwent prophylactic total thyroidectomy at the age of 5 years and 6 months; surgical pathology showed CCH. Only this patient experienced increased serum calcitonin postoperatively; thus, complete total thyroidectomy was performed by an experienced endocrine surgeon and pediatric surgeons. At the operation time his height was $105.4 \mathrm{~cm}$ and body weight $18.05 \mathrm{~kg}$. His preoperative thyroglobulin level was 42.0$106.4 \mathrm{ng} / \mathrm{mL}$. After total thyroidectomy, the thyroglobulin level was $0.55-2.41 \mathrm{ng} / \mathrm{mL}$ (his institutional normal thyroglobulin level was $0.0-32.7 \mathrm{ng} / \mathrm{mL}$.

Patient no. 2 was the only patient whose preoperative calcitonin level was measured via ECLIA. An MTC tumor with a maximum diameter of $2 \mathrm{~mm}$ was found; it was cured biochemically following surgery. This case was reported in detail by Abe et al. [22].

\section{ATA moderate-risk patients}

All patients in this category who underwent surgery had prophylactic resection before without increased 
Table 2: Incidence of any complications after thyroidectomy according to age.

\begin{tabular}{|c|c|c|c|c|}
\hline \multirow[b]{3}{*}{ Type of complication } & \multicolumn{4}{|c|}{ Children with any postoperative complications } \\
\hline & \multirow[b]{2}{*}{ Total (\%) } & \multicolumn{3}{|c|}{ Age (years) } \\
\hline & & $3-6$ & $7-12$ & $13-18$ \\
\hline Transient RLN palsy & 2 of $12(17)$ & 0 of $3(0)$ & 1 of $7(14)^{n}$ & 1 of $2(50)^{\S}$ \\
\hline Transient hypoparathyroidism & 2 of $12(17)$ & 1 of $3(33)^{\S}$ & 1 of $7(14)^{\pi}$ & 0 of $2(0)$ \\
\hline Permanent hypoparathyroidism & 4 of $12(33)$ & 0 of $3(0)$ & 3 of $7(43)^{\uparrow, *}$ & 1 of $2(50)^{*}$ \\
\hline Any complication & 8 of $12(67)$ & 1 of $3(33)$ & 5 of $7(71)$ & 2 of $2(100)$ \\
\hline
\end{tabular}

qWith central dissection.

*With central and lateral dissection.

\$Thyroidectomy.

Abbreviations: RLN, recurrent laryngeal nerve.

calcitonin levels determined via RIA. The cumulative incidence of pathological onset or first calcitonin elevated timing reached $50 \%$ at the age of 8 years (11 years upon adjustment for competing risks) and $100 \%$ at the age of 17 years (Figure 2). Because the high calcitonin levels in patient 12 further increased from $4.9 \mathrm{pg} / \mathrm{mL}$ to $10.8 \mathrm{pg} / \mathrm{mL}$ (basal value) and from $120 \mathrm{pg} / \mathrm{mL}$ to $338 \mathrm{pg} / \mathrm{mL}$ (stimulated value) at the age of 3 years 4 months, we considered the high calcitonin levels at the age of 2 years to be the first elevated calcitonin timing.

\section{Surgical and postoperative complications}

Postoperative complications are shown in Tables 2 and 3. Transient RLN palsy occurred in a moderate-risk patient aged 17 years (patient no. 6) and a high-risk patient aged 10 years (patient no. 3). Two high-risk patients (aged 5 and 9 years; no. 1 and 2, respectively) experienced transient hypoparathyroidism (Table 2). Hypoparathyroidism was the only permanent complication $(n=4$; patients no. $17,19,20$, and 21); it occurred only in the highest-risk patients who required lymphadenectomy (Table 3). No permanent complications were observed in patients who did not undergo cervical lymphadenectomy or were aged
5-6 years. The incidence of permanent complications increased with age and as the area of the neck lymphadenectomy widened. As shown in Figure 3, patients were referred to many different surgeons by the physician in charge. The surgery department showed no correlation with postoperative complications.

\section{Discussion}

We confirmed the several tendencies in Japanese pediatric MEN2: those of therapy policy variations in each facility and higher age at thyroidectomy than that recommended by the ATA. There were no permanent complications due to prophylactic thyroidectomy, but there were many transient surgical complications. Moreover, there was a high proportion of surgical complications among advanced MEN2B cases with lymphadenectomy. In order to solve these problems, it is necessary to discuss whether prophylactic thyroidectomy at low age will reduce surgical complications and improve the probability of recurrence. It is also necessary to consider whether there is merit in cost.

First, regarding operative complications, few studies have reported on postoperative complications of

Table 3: Permanent complications after surgery by extent of surgery according to age.

\begin{tabular}{|c|c|c|c|c|}
\hline \multirow[b]{3}{*}{ Extent of surgery } & \multicolumn{4}{|c|}{ Children with permanent postoperative hypoparathyroidism } \\
\hline & \multirow[b]{2}{*}{ Total (\%) } & \multicolumn{3}{|c|}{ Age, years } \\
\hline & & $3-6$ & $7-12$ & $13-18$ \\
\hline Thyroidectomy & 0 of $3(0)$ & 0 of $1(0)$ & 0 of $1(0)$ & 0 of $1(0)$ \\
\hline With central dissection & 1 of $6(17)$ & 0 of $2(0)$ & 1 of $4(25)$ & \\
\hline With central and lateral dissection & 3 of $3(100)$ & & 2 of $2(100)$ & 1 of $1(100)$ \\
\hline Any surgery & 4 of $12(33)$ & 0 of $3(0)$ & 3 of $7(43)$ & 1 of $2(50)$ \\
\hline
\end{tabular}




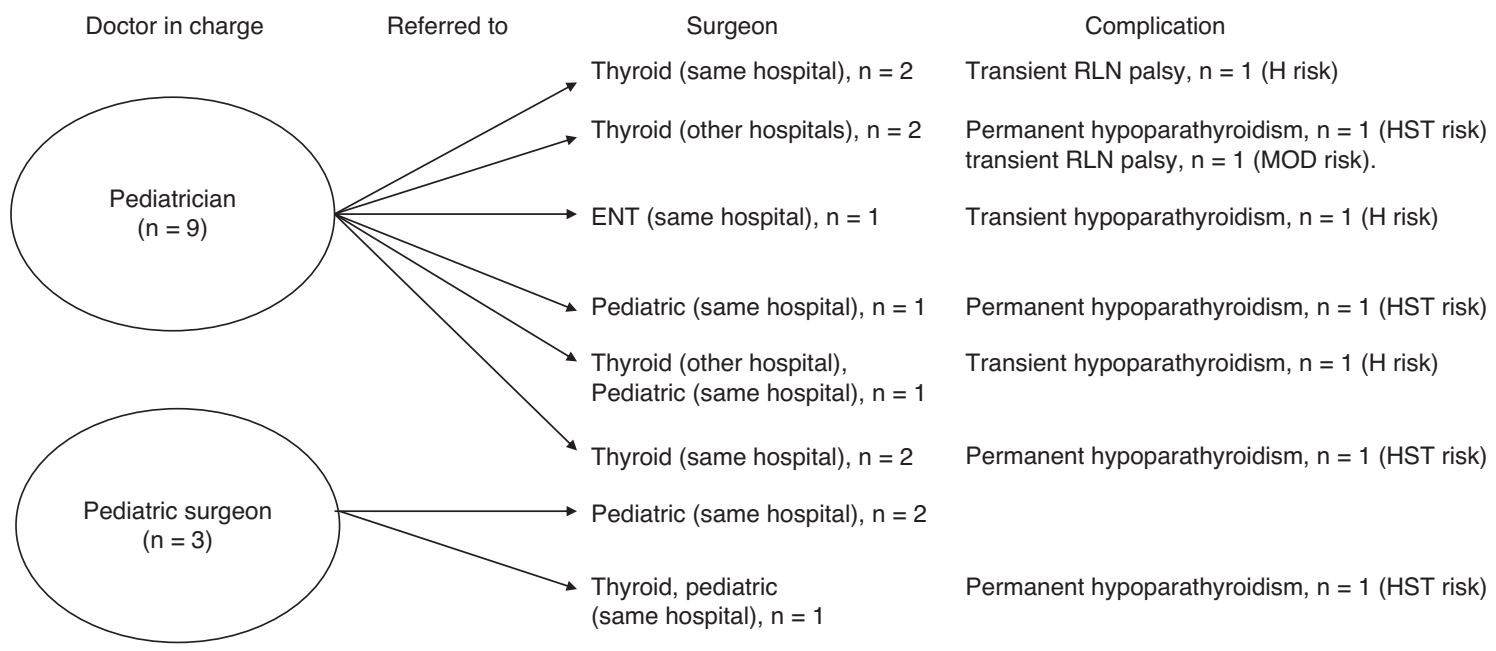

Figure 3: Types of referrals from physicians in charge to surgeons and postoperative complications.

Own: Surgery was performed at the same institution (the physician in charge referred the patient to a surgeon within the same facility for surgery). Other: Patient was referred to a surgeon at another facility.

prophylactic thyroidectomy in children (Table 4) [7, 9, 23, 24], revealing a higher risk of postoperative complications when neck lymphadenectomy was performed at a young age. In those studies, and ours, permanent RLN palsy occurred in only a 1-year-old boy [7] out of 347 patients $(0.3 \%)$. Permanent hypothyroidism occurred in $27 / 347$ patients (7.8\%). There was no surgery-related death. Generally, the parathyroid glands of children younger than 5 years are translucent and small; postoperative complications, including hypoparathyroidism, are more common with pediatric thyroid surgery when compared to those in adults [6]. However, adult Japanese MEN2 patients who underwent thyroidectomy with $94.9 \%$ neck lymphadenectomy also had a high operative complication rate: permanent RLN palsy 11/330 (3.3\%), permanent hypothyroidism 74/327 (22.6\%) [11]; 80\% of MEN2B patients of higher age in our study had permanent hypoparathyroidism. These suggest that clinically apparent disease requiring aggressive surgery with neck lymphadenectomy is associated with a high rate of permanent complications.

The ATA guidelines recommend that patients should be referred to experienced surgeons to decrease the rate of postoperative complications $[5,6]$. It is noteworthy that facilities with a high number of pediatric thyroid surgeries exhibited no permanent surgical complications [9]. The present study analyzed data from multiple facilities at which thyroid surgeries were not frequently performed in children. The incidence of transient RLN palsy was $1.8 \%$ in high-volume facilities; in contrast, it was $28.6 \%(2 / 7$ patients with MEN2A) in our study [9]. Another nationwide report described a high proportion of permanent hypoparathyroidism in 15/79 patients (19.0\%) [24].
Second, regarding recurrence, we believe that operations for children in high-volume facilities also reduce recurrence. In reports where the definition of prophylactic thyroidectomy was closest to that of the ATA and where age was considered in high-volume facilities, the rate of recurrence or residual disease was $0.7-5 \%[7,9]$. On the other hand, a nationwide survey that included cases with delayed surgery had $62 \%$ recurrence or residual disease proportion [24]. Of course, it was obvious that the definitions were different in that report. The data regarding prophylactic thyroidectomy among pediatric patients with MEN2 are shown in Table 4 [7, 9, 23, 24]. Patients with suspected recurrence or residual disease all had biochemical recurrence or were at the residual disease stage, except for one MEN2B patient with advanced MTC [24]. Notably, in hereditary MTC, recurrence was observed in both highand moderate-risk patients aged $>8$ years, even if MTC was not in the advanced stage [23].

Our study included a patient with a high-risk C634Y mutation who had elevated serum calcitonin levels after undergoing total thyroidectomy at 5 years of age with $\mathrm{CCH}$ in pathology. Detectable thyroglobulin level may indicate remnant thyroid tissue in this patient. However, because micrometastases can occur during an early stage in MTC, recurrence or residual disease cannot also be denied. The recurrence proportion in hereditary MTC is $4 \%$ among cases with tumor size $<2 \mathrm{~mm}$ and node negative at surgery [1]. Hereditary MTC has an estimated annual growth of $0.4-0.5 \mathrm{~mm}$ [25]. Accordingly, at least after the elevation of calcitonin, surgery should not be postponed. MTC-related death at 12 years has been reported in moderate-risk patients [26]. The ATA states that delaying surgery after 


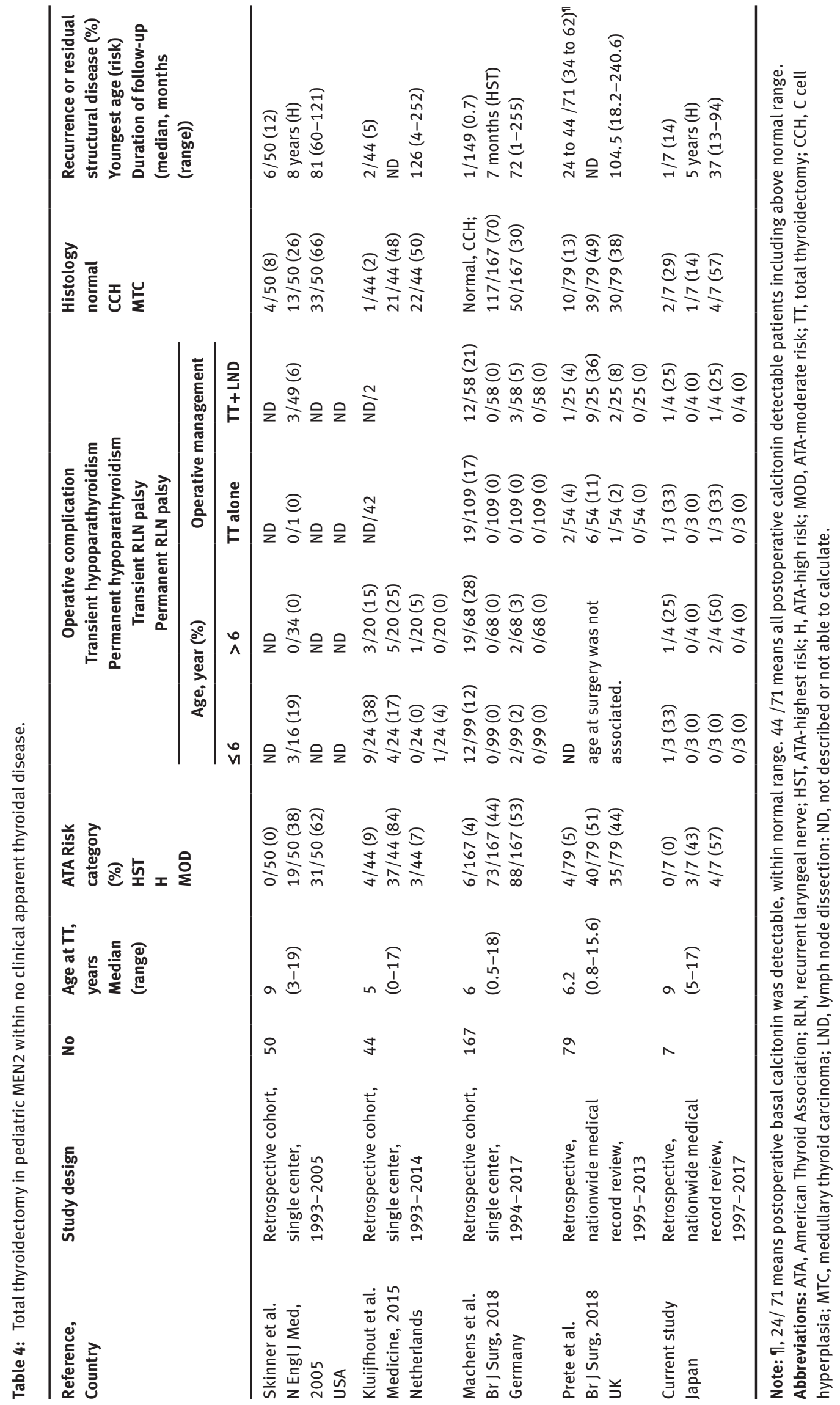


disease onset increases the risk of recurrence. However, in patients aged $\leq 3$ years, calcitonin levels are physiologically high; thus, an early determination of disease onset is challenging [20]. Moreover, unexpected postoperative complications can occur [7]. Thyroid hormones substantially affect an individual's growth and development [27], particularly in children; thus, cautious judgment is necessary. Because metastatic MTC is extremely rare in patients with MEN2A aged $\leq 3$ years, the ATA does not recommend surveillance in this patient population.

Lastly, we should solve the cost problem for children. In Japan, most medical expenses are covered by the universal healthcare insurance system. However, prophylactic surgery is not covered. This may be one reason why most children aged $<10$ years do not undergo prophylactic resection [10]. This may have influenced the mean age at MTC-related death in patients with MEN2, i.e. 53 years [28], causing economic loss.

There is an obvious cost benefit for preventive removal. About 30 MEN2 patients were born yearly in Japan. If the gene mutation is already known, the cost of 1 exon is $\$ 350$ ( $1 \$=110$ JPY), and the total cost for 30 persons with autosomal dominant siblings without genetic mutation is about $\$ 10,820$. This is not high considering unnecessary surveillance. In addition, the fee for levothyroxine $100 \mu \mathrm{g}$ per day per year comes to $\$ 36$ per person. When hypoparathyroidism occurs, the cost of $0.5 \mu \mathrm{g}$ per day of alfacalcidol per year comes to $\$ 198$ per person. The monthly per capita expenditure for one person using vandetanib in the event of a recurrence is $\$ 12,236.40$ [29].

In countries similar to Japan that have cost problems with prophylactic thyroidectomy, early calcitonin level-based thyroidectomy not based on age is needed to reduce surgical delay and postoperative complications and improve prognosis in pediatric MEN2. This is because early thyroidectomy may improve the timing of surgery with a large heterogeneity in the age of onset and aggressiveness of MTC. A previous study reported that prognosis after disease onset is similar in high- and moderate-risk patients [30], even in MEN2B [31]. The penetration rate of MTC among individuals aged 50 years is $83 \%$ in high-risk patients [32], whereas it varies among those with a familial history of identical genetic mutations [22, 33].

Our study showed that, in patients with MEN2A, using ECLIA-based calcitonin measurements to speculate the onset allowed for earlier recognition of the disease when compared to the results of other studies. In our study, the cumulative incidence of CCH or MTC or first increased serum calcitonin reached $50 \%$ at the age of 5 years and $100 \%$ at the age of 9 years and reached $50 \%$ at the age of 8 years and $100 \%$ in moderate-risk patients aged $\geq 17$ years. A study in high-risk MEN2A patients who underwent surgery reported that $\mathrm{CCH}$ and MTC occurred at the ages of 6.9 years and 10.1 years on average, respectively [2]. For moderate-risk, another report showed that the cumulative incidence of MTC reached 50\% in moderate-risk patients aged 13-18 years [9]. Reasons potentially explaining these differences are as follows. First, our study could not distinguish CCH or MTC in patients who did not undergo surgery. Second, there were differences in patient risks that were included. In our study, moderate-risk included only extracellular $R E T$ mutations that were classified as level B in the previous ATA guideline [4]. However, there may not be a strong selection bias. For example, in the German series, the prevalence of risk was determined as level $\mathrm{B}$, that is higher risk in moderate-risk 144/567 (25.4\%) or level A 163/567 (28.7\%) [34], whereas in the Japan series, risk was level B 144/505 (28.5\%) and level A 45/505 (8.9\%) [11]. Lastly, as this study included patients without surgery, there is a possibility of false positives.

A study of medullary thyroid microcarcinoma in adults reported that lymph node metastases in the neck were observed in patients with calcitonin levels of $10-40 \mathrm{pg} / \mathrm{mL}$, measured via the solid two-site immunoradiometric calcitonin assay [13]. Meanwhile, no recurrence was observed among children whose preoperative calcitonin level was $<30 \mathrm{pg} / \mathrm{mL}$, as measured via immunoradiometric assays during long-term follow-up [35]. In our study, disease onset possibility was recognized starting at very low calcitonin levels $(<10 \mathrm{pg} / \mathrm{mL})$. Thus, even in cases in which total thyroidectomy was performed at a higher age, the indication for surgery, i.e. the prevention of recurrence was still within that stipulated by the ATA.

The present study has many limitations, including its retrospective design and the small number of included cases due to the rarity of the disease. Moreover, the data were only collected from pediatricians and pediatric surgeons; thus, selection bias was unavoidable. The degree of progression in patients who did not undergo surgery was unclear, and the number of cases in which preoperative calcitonin levels were measured via the ECLIA was small. The calcium stimulation test is the only test used in Japan that is nonspecific compared to the pentagastrin stimulation test. Our study also included patients whose detailed clinical histories and prognoses were unavailable, due to changing hospitals or for other reasons, and the follow-up period was short; thus, the long-term prognosis related to recurrence could not be analyzed.

In conclusion, prophylactic thyroidectomy may be advantageous in patients with MEN2 as it can achieve a balance between a reduction of postoperative 
complications and the risk of recurrence. However, considering the large heterogeneity in hereditary MTC, measuring calcitonin levels via the ECLIA can provide a clinical basis for early thyroidectomy in collaboration with various medical specialties and in consideration of the country's medical system.

Acknowledgments: We thank the following JSPE members for their invaluable support: Tsutomu Ogata, Yasuko Fujisawa, Takashi Hamajima, Yuki Abe, Mika Inoue, Makiko Tachibana, Takuo Kubota, Noriyuki Namba, Masatsune Itoh, Yukihiro Hasegawa, Aya Shimada, Reiko Horikawa, Chikahiko Numakura, Ikuma Fujiwara, Hiroyo Mabe, Takeshi Usui, and Satoshi Narumi. We would also like to thank the members of the Research Group for "Comprehensive Study and Seamless Guidelines" on rare and intractable gastrointestinal disease from childhood, supported by the Ministry of Health, Labor and Welfare of Japan: Tamotsu Kobayashi, Koichi Saito, Yuhki Arai, Kazuhiko Jinno, Yasutsugu Chinen, Mahoko Furujo, Atsushi Yoden, Emiri Kaji, Sotaro Mushiake, and Tomoaki Taguchi. Ethics approval was obtained with the assistance of Eiko Nagata. Statistics support was provided by Eisaku Okada. Last, we thank the MEN consortium of Japan members Akihiro Sakurai, Shinichi Suzuki, and Shinya Uchino for providing relevant information.

Author contributions: Rie Matsushita created the research plan and drafted as well as revised the manuscript. Kenji Ihara, Tomonobu Hasegawa and Keiichi Ozono assessed and revised the research plan. Keisuke Nagasaki, Tadayuki Ayabe, Youko Miyoshi, Saori Kinjyo, Hidenori Haruna, and Shinobu Ida collected the data and performed the analyses. Kanshi Minamitani led the team and was involved in all phases of the study. We would like to thank Editage (www.editage.jp) for English language editing.

Research funding: None declared.

Employment or leadership: None declared.

Honorarium: None declared.

Competing interests: The authors report no conflicts of interest regarding this work.

\section{References}

1. Machens A, Dralle H. Biological relevance of medullary thyroid microcarcinoma. J Clin Endocrinol Metab 2012;97:1547-53.

2. Machens A, Niccoli-Sire P, Hoegel J, Frank-Raue K, van Vroonhoven TJ, et al. Early malignant progression of hereditary medullary thyroid cancer. New Engl J Med 2003;349:1517-25.

3. Brandi ML, Gagel RF, Angeli A, Bilezikian JP, Beck-Peccoz P, et al. Guidelines for diagnosis and therapy of MEN type 1 and type 2 . J Clin Endocrinol Metab 2001;86:5658-71.
4. Kloos RT, Eng C, Evans DB, Francis GL, Gagel RF, et al. Medullary thyroid cancer: management guidelines of the American Thyroid Association. Thyroid 2009;19:565-612.

5. Wells SA, Jr., Asa SL, Dralle H, Elisei R, Evans DB, et al. Revised American Thyroid Association guidelines for the management of medullary thyroid carcinoma. Thyroid 2015;25:567-610.

6. Tuggle CT, Roman SA, Wang TS, Boudourakis L, Thomas DC, et al. Pediatric endocrine surgery: who is operating on our children? Surgery 2008;144:869-77.

7. Kluijfhout WP, van Beek DJ, Verrijn Stuart AA, Lodewijk L, Valk GD, et al. Postoperative complications after prophylactic thyroidectomy for very young patients with multiple endocrine neoplasia type 2: retrospective cohort analysis. Medicine (Baltimore) 2015;94:e1108.

8. Machens A, Elwerr M, Thanh PN, Lorenz K, Schneider R, et al. Impact of central node dissection on postoperative morbidity in pediatric patients with suspected or proven thyroid cancer. Surgery 2016;160:484-92.

9. Machens A, Elwerr M, Lorenz K, Weber F, Dralle H. Long-term outcome of prophylactic thyroidectomy in children carrying RET germline mutations. Br J Surg 2018;105:e150-7.

10. Uchino S. Presymptomatic diagnosis and timing of prophylactic total thyroidectomy in pediatric medullary thyroid carcinoma. Saishin Igaku 2013;68:1867-73 (in Japanese).

11. Uchino S. [Multiple endocrine neoplasia type 2 in Japan: largescale analysis of data from the MEN consortium of Japan]. Nihon Geka Gakkai Zasshi 2012;113:362-7 (in Japanese).

12. Hogan AR, Zhuge Y, Perez EA, Koniaris LG, Lew JI, et al. Pediatric thyroid carcinoma: incidence and outcomes in 1753 patients. J Surg Res 2009;156:167-72.

13. Machens A, Schneyer U, Holzhausen HJ, Dralle H. Prospects of remission in medullary thyroid carcinoma according to basal calcitonin level. J Clin Endocrinol Metab 2005;90:2029-34.

14. Kahaly GJ, Algeciras-Schimnich A, Davis TE, Diana T, Feldkamp J, et al. United States and European multicenter prospective study for the analytical performance and clinical validation of a novel sensitive fully automated immunoassay for calcitonin. Clin Chem 2017;63:1489-96.

15. Kitagawa W, Sugino K, Ito K. New assay of calcitonin measurements. Naibunpitu Koujyousen Geka Gakkai Zassi 2017;34:32-6 (in Japanese).

16. Wells SA, Jr., Baylin SB, Gann DS, Farrell RE, Dilley WG, et al. Medullary thyroid carcinoma: relationship of method of diagnosis to pathologic staging. Ann Surg 1978;188:377-83.

17. Kihara M, Miyauchi A, Kudo T, Hirokawa M, Miya A. Reference values of serum calcitonin with calcium stimulation tests by electrochemiluminescence immunoassay before/after total thyroidectomy in Japanese patients with thyroid diseases other than medullary thyroid carcinoma. Endocr J 2016;63:627-32.

18. Kihara M, Miyauchi A, Kudo T, Hirokawa M, Miya A. Serum calcitonin reference values for calcium stimulation tests by electrochemiluminescence immunoassay in Japanese men with non-medullary thyroid carcinoma. Surg Today 2018;48:223-8.

19. Mian C, Perrino M, Colombo C, Cavedon E, Pennelli G, et al. Refining calcium test for the diagnosis of medullary thyroid cancer: cutoffs, procedures, and safety. J Clin Endocrinol Metab 2014;99:1656-64.

20. Castagna MG, Fugazzola L, Maino F, Covelli D, Memmo S, et al. Reference range of serum calcitonin in pediatric population. J Clin Endocrinol Metab 2015;100:1780-4. 
21. Basuyau JP, Mallet E, Leroy M, Brunelle P. Reference intervals for serum calcitonin in men, women, and children. Clin Chem 2004;50:1828-30.

22. Abe Y, Tsukano S. Detection of early stage medullary thyroid carcinoma by measuring serum calcitonin using an electro chemiluminescence immuno-assay: a case report of a young Japanese woman with a high-risk RET mutation. Clin Pediatr Endocrinol 2017;26:243-9.

23. Skinner MA, Moley JA, Dilley WG, Owzar K, Debenedetti MK, et al. Prophylactic thyroidectomy in multiple endocrine neoplasia type 2A. New Engl J Med 2005;353:1105-13.

24. Prete FP, Abdel-Aziz T, Morkane C, Brain C, Kurzawinski TR. Prophylactic thyroidectomy in children with multiple endocrine neoplasia type 2. Br J Surg 2018;105:1319-27.

25. Machens A, Lorenz K, Dralle H. Progression of medullary thyroid cancer in RET carriers of ATA class A and C mutations. J Clin Endocrinol Metab 2014;99:E286-92.

26. Frohnauer MK, Decker RA. Update on the MEN 2A c804 RET mutation: is prophylactic thyroidectomy indicated? Surgery 2000;128:1052-7.

27. Minamitani K, Inomata $\mathrm{H}$. Neonatal screening for congenital hypothyroidism in Japan. Pediatr Endocrinol Rev 2012; 10(Suppl 1):79-88.

28. Imai T, Uchino S, Okamoto T, Suzuki S, Kosugi S, et al. High penetrance of pheochromocytoma in multiple endocrine neoplasia 2 caused by germ line RET codon 634 mutation in Japanese patients. Eur J Endocrinol 2013;168:683-7.
29. Cooper MR, Yi SY, Alghamdi W, Shaheen DJ, Steinberg M. Vandetanib for the treatment of medullary thyroid carcinoma. Ann Pharmacother 2014;48:387-94.

30. Voss RK, Feng L, Lee JE, Perrier ND, Graham PH, et al. Medullary thyroid carcinoma in MEN2A: ATA moderate- or high-risk RET mutations do not predict disease aggressiveness. J Clin Endocrinol Metab 2017;102:2807-13.

31. Raue F, Dralle H, Machens A, Bruckner T, Frank-Raue K. Longterm survivorship in multiple endocrine neoplasia type $2 \mathrm{~B}$ diagnosed before and in the new millennium. J Clin Endocrinol Metab 2018;103:235-43.

32. Milos IN, Frank-Raue K, Wohllk N, Maia AL, Pusiol E, et al. Age-related neoplastic risk profiles and penetrance estimations in multiple endocrine neoplasia type $2 \mathrm{~A}$ caused by germ line RET Cys634Trp (TGC > TGG) mutation. Endocr Relat Cancer 2008;15:1035-41.

33. Bihan H, Baudin E, Meas T, Leboulleux S, Al Ghuzlan A, et al. Role of prophylactic thyroidectomy in RET 790 familial medullary thyroid carcinoma. Head Neck 2012;34:493-8.

34. Machens A, Lorenz K, Weber F, Dralle H. Genotype-specific progression of hereditary medullary thyroid cancer. Hum Mutat 2018;39:860-9.

35. Rohmer V, Vidal-Trecan G, Bourdelot A, Niccoli P, Murat A, et al. Prognostic factors of disease-free survival after thyroidectomy in 170 young patients with a RET germline mutation: a multicenter study of the Groupe Francais d'Etude des Tumeurs Endocrines. J Clin Endocrinol Metab 2011;96:E509-18. 